\title{
Coherent tunnel repolarization of a hydrogen bonded chain
}

\author{
P.M.Tomchuk, S.P.Luk'yanets \\ Institute of Physics, the National Academy of Sciences of Ukraine, \\ 46 Nauky Av., UA-252650 Kyiv-22, Ukraine
}

Received April 24, 1998

\begin{abstract}
In the present paper we suggest a possible mechanism of repolarization of a short hydrogen bonded chain. This mechanism is based on the coherent tunnel reorientation of ionic groups and is additional to the other one that is realized due to the coherent tunnel repolarization of the hydrogen bonds described in the previous work (J. Mol. Struct. 416, 161 (1997)). With the aid of the instanton method the tunnelling frequency of this reorient transition is calculated in the WKB approximation. The tunnelling frequency of such a flip-flop process does not depend on temperature, and this effect can be observed at low temperatures when tunnelling chain repolarization prevails over the thermal activation one.
\end{abstract}

Key words: hydrogen bond, macroscopic quantum tunneling, macroscopic quantum coherene, proton transfer

PACS: $64.60 . \mathrm{Cn}, 66.30 . \mathrm{Lw}$

\section{Introduction}

Many basic properties of physical and biological objects are governed by hydrogen bonds. Ferroelectric, structural phase transitions, ion and proton transport in such systems are related to proton motion at a hydrogen bond and reorientation of the ionic groups [1]-[9]. The short hydrogen-bonded chains are one of the main proton channel elements of proton-conducting biomembranes [10,11]. It is well known $[10,12-17]$, that this chain has a great polarisability which fluctuates between two opposite directions. Many optical and kinetic properties of H-bonded chains are defined by different mechanisms of the polarization oscillations. Thus, the polarization modes themselves ensure the possibility of a polaron to be involved into the proton transport along the chain [18], and transport of the ionic and orientational defects can be considered, in some cases, as a result of a chain repolarization (see e.g. $[6,7,20,21]$ ). Here we propose a possible mechanism of repolarization of short hydrogen bonded chains based on macroscopic quantum tunnelling [22-26]. This mechanism corresponds to the tunnelling of a macroscopic variable through 
a barrier between two minima of the effective potential of a macroscopic system. In particular, such effect is observed in a small ferromagnetic particle whose size is smaller than the characteristic one of a magnetic domain [25,27,28]. The magnetic moment of a one-domain particle has several equilibrium directions of easy magnetization determined by the crystalline anisotropy and shape of the particle. The oscillation induced switching of the particle's magnetic moment between these directions does not disappear with temperature decreasing [29]. In other words, at low temperature the particle magnetic moment tunnels between different energy minima. The repolarization of the hydrogen bonded chain can be realized as an effect due to the repolarization of the hydrogen bonds $\mathrm{O}-\mathrm{H} \ldots \mathrm{O} \leftrightarrow \mathrm{O} \ldots \mathrm{H}-\mathrm{O}$ ) and/or due to reorientation of the ion groups $\mathrm{O}-\mathrm{H} \ldots \mathrm{O}-\mathrm{H} \leftrightarrow \mathrm{H}-\mathrm{O} \ldots \mathrm{H}-\mathrm{O}$. One of the possible mechanisms of repolarization is a soliton mechanism of an overbarrier reconstruction of a chain $[6,7,20,21]$. As it was shown in [26] in the framework of the pseudo-spin representation for the proton subsystem, the polarization vector of a short H-bonded chain can tunnel, as a single domain, between two opposite states $\mathrm{O}-\mathrm{H} . . . \mathrm{O}-\mathrm{H} \ldots \mathrm{O}$ and $\mathrm{O} \ldots \mathrm{H}-\mathrm{O} \ldots \mathrm{H}-\mathrm{O}$ which have the same energy. These transitions take place by way of a coherent tunnel repolarization of chain hydrogen bonds and do not lead to the formation of defects as it is in the case of the successive overor through-barrier repolarization of each hydrogen bond.

In the present paper we show that a tunnel repolarization of a short one-domain $\mathrm{H}$-bonded chain can be due not only to the proton motion along the hydrogen bond [26] but can occur due to the coherent tunnel motion of protons around heavy ions (coherent tunnel reorientation of the hydroxyl group: O-H...O-H... $\leftrightarrow$ $\mathrm{H}-\mathrm{O} . . . \mathrm{H}-\mathrm{O} \ldots$... . The latter becomes possible when protons are tightly bound with the ion groups, so that the probability of proton transfer processes along the hydrogen bond is lower than that of orientation effects of the group O-H. To illustrate the feasibility of such transitions it is sufficient to study the simplest models of orientation oscillations of the ionic groups of a hydrogen bonded chain $[7,8,20,21]$. In the two-level approximation, these models can be reduced to the model of an easy-axes ferromagnetic with a transverse external field for which the effect of a macroscopic quantum coherence was considered in detail (see, e.g., [16-20]). The authors of [21] proposed a model of the orientation kink-defect in a quasi onedimensional ice crystal. The main dynamic variable in that case was the angle between the direction of an $\mathrm{O}-\mathrm{H}$ bond and that of the water molecules chain. The potential energy of this system is determined by the interaction between the nearest water molecules and the two-well potential, whose minima correspond to the equilibrium orientations (... O-H... and ...H-O...) of water molecules in the chain. If we neglect the excited states and consider a 2-level scheme, then, in a pseudo-spin representation, the secondary quantised Hamiltonian of such a system is known to be reduced to the model of easy-axes ferromagnetic with a transverse external field [5]. To estimate the frequency of coherent orientation tunnel transitions we consider another model [8] that takes into account both the proton dynamics of hydrogen bonds and the reorientation processes of $\mathrm{O}-\mathrm{H}$ groups. This model admits two limiting cases of particular interest for the study. In the first case the coherent 
tunnel repolarization of one-domain chain is determined by the hydrogen bonds repolarization and was described in [26]. In the second case, studied below, the repolarization is determined by a hydroxyl-group reorientation that takes place through the proton motion around an ion group.

\section{Pseudo-spin realization of the orientation molecule motion of one-domain H-bonded chain}

We will consider a quasi one-dimensional hydrogen bonded chain, schematically presented as A-H...A-H...A-H... . Suppose that two different configurations of the chain H-A...H-A... and A-H...A-H... have the same energy. The purpose of the present paper is to show that transitions of the short chain between these configurations can occur through the coherent tunnel reorientation of groups A-H. The motion of a proton in an H-bond can be described in terms of a two-well potential. Using a two-level approximation, i.e. taking into account only two lowest proton levels, one may introduce Fermi-operators $a_{r, k}^{+}\left(a_{r, k}\right)$ and $a_{l, k}^{+}\left(a_{l, k}\right)$ of proton creation and annihilation in the right and left wells of a $k$-th hydrogen bond. As shown in [8], orientation transitions of A-H groups can be described in terms of a proton subsystem as a pseudo-tunnel effect. The Hamiltonian of this model can be written as [8]:

$$
H=H_{0}+H_{t}+H_{r}+H_{c}
$$

where

$$
H_{0}=\sum_{k} w^{\prime}\left(1-n_{r, k}\right)\left(1-n_{l, k+l}\right)+w n_{r, k} n_{l, k+1}+\varepsilon\left(1-n_{r, k}\right) n_{l, k+l}+\varepsilon n_{r, k}\left(1-n_{l, k+1}\right)
$$

determines a short-range interaction between protons at the nearest hydrogen bonds; $w^{\prime}, w, \varepsilon$ are the energies of proton configurations in minima of the potential near an ionic group; $n_{r, k}\left(n_{l, k}\right)$ is an operator of the proton number in the right (left) well of a $k$-th $\mathrm{H}$-bond. The term $H_{t}$ describes a tunnel transition between two proton states in the hydrogen bond:

$$
H_{t}=-\eta \Omega_{0} \sum_{k} a_{l, k}^{+} a_{r, k}+a_{r, k}^{+} a_{l, k}
$$

where $\Omega_{0}$ is the tunneling frequency.

In such an approach, orientation transitions of the ionic groups $(\mathrm{A}-\mathrm{H}) \leftrightarrow(\mathrm{H}-\mathrm{A})$, can be described as a pseudo-tunnel effect with frequency $\Omega_{r}$ :

$$
H_{t}=-\eta \Omega_{r} \sum_{k} a_{r, k}^{+} a_{l, k+l}+a_{l, k+1}^{+} a_{r, k} .
$$

The term

$$
H_{0}=U \sum_{k} n_{r, k} n_{l, k}+V \sum_{k}\left(1-n_{r, k}\right)\left(1-n_{l, k}\right)
$$


corresponds to the Coulomb repulsion energy between two protons in the same hydrogen bond and that of free electron pairs, with protons missing [8,9,30]. For simplicity, we regard that all hydrogen bonds are equivalent and the chain obeys the periodical boundary condition.

Consider the situation when a proton is strongly connected with a heavy atom, so that one can neglect the tunnel proton transitions along the hydrogen bond in comparison with the reorientation effects of the groups A-H. It enables us to suppose that only one proton is localized near every heavy atom, i.e. $n_{r, k}+n_{l, k}=1$, and we can introduce the notion of a pseudospin (see, e.g., [5]):

$$
\begin{aligned}
s_{k}^{x} & =\frac{1}{2}\left(a_{r, k}^{+} a_{l, k+1}+a_{l, k+1}^{+} a_{r, k}\right), \\
s_{k}^{y} & =\frac{1}{2}\left(a_{r, k}^{+} a_{l, k+1}-a_{l, k+1}^{+} a_{r, k}\right), \\
s_{k}^{z} & =\frac{1}{2}\left(a_{r, k}^{+} a_{r, k}-a_{l, k+1}^{+} a_{l, k+1}\right),
\end{aligned}
$$

where $z$ is a pseudospin component that corresponds to the orientation of the $k$-th A-H group, the $x$-th component is an operator of the tunnel reorientation and the $y$ component is an operator of the local current. Using our assumptions from equation (1-5) and equation (6) we get a reduced Hamiltonian for the orientation motion of an ionic group in the H-bonded chain

$$
H^{\prime}=-\hbar \Omega \sum_{k} s_{k}^{x}+J \sum_{k} s_{k-1}^{z} s_{k}^{z}
$$

where $\Omega=2 \Omega_{r}, J=U+V$, and the equations of motion for operator $\boldsymbol{s}_{k}$ are given by

$$
\begin{aligned}
\hbar \frac{\mathrm{d} s_{k}^{x}}{\mathrm{~d} t} & =J\left(s_{k}^{y} s_{k+1}^{z}+s_{k-1}^{z} s_{k}^{y}\right), \\
\hbar \frac{\mathrm{d} s_{k}^{y}}{\mathrm{~d} t} & =\hbar \Omega s_{k}^{z}-J\left(s_{k}^{y} s_{k+1}^{z}+s_{k-1}^{z} s_{k}^{y}\right), \\
\hbar \frac{\mathrm{d} s_{k}^{x}}{\mathrm{~d} t} & =-\hbar \Omega s_{k}^{y} .
\end{aligned}
$$

Thus, in a two-level approximation the Hamiltonian of orientation oscillations of the ionic group in the hydrogen bonded chain, equation (7), corresponds to the model of easy-axis ferromagnetic in the transverse external field $2 \hbar \Omega_{r}$. As it has been specified above, we can obtain $H^{\prime}$ by quantizing the classical model [21] and determine the parameters $\Omega, J$ in an explicit form.

In the mean field approximation the motion equation of the average value of pseudospin $\boldsymbol{S}_{k}=\left\langle\boldsymbol{s}_{k}\right\rangle$ has the following form [5]:

$$
\hbar \frac{\mathrm{d} \boldsymbol{S}_{k}}{\mathrm{~d} t}=\boldsymbol{S}_{k} \times \boldsymbol{F}_{k},
$$

where

$$
\boldsymbol{F}_{k}=-\frac{\partial\left\langle H^{\prime}\right\rangle}{\partial \boldsymbol{S}_{k}}
$$


Equations (9) and (10) are equivalent to the equations of free classical precession of a pseudospin round the momentary value of the mean field $\boldsymbol{F}_{k}$. In our case the classical value of pseudospin $\boldsymbol{S}_{k}$ corresponds to the orientation of an A-H group and can be rewritten in terms of its polarization vector $\boldsymbol{P}_{k}=2 \boldsymbol{S}_{k} d$ (where $d$ is a dipole momentum of an A-H group).

Now we take into account the fact that the short H-bonded chain is a single orientation (or polarization) domain. Following [25,26], one can set $\boldsymbol{S}_{k}=\boldsymbol{S}$. Then equation (9) is given by

$$
\hbar \frac{\mathrm{d} \boldsymbol{S}}{\mathrm{d} t}=-\boldsymbol{S} \times \frac{\partial E}{\partial \boldsymbol{S}}
$$

where $E$ is the orientational anisotropy energy

$$
E=-\hbar \Omega S^{x}-J\left(S^{z}\right)^{2}
$$

Vector $\boldsymbol{S}$ characterises the orientation (or polarization) of a one-domain chain. In the spherical coordinate system vector $\boldsymbol{S}=(S \sin \theta \cos \phi, S \sin \theta \sin \phi, S \cos \theta)$ and equation (11) reduce to

$$
\begin{aligned}
\hbar S \frac{\mathrm{d} \theta}{\mathrm{d} t} \sin \theta & =\frac{\partial E}{\partial \phi}, \\
\hbar S \frac{\mathrm{d} \phi}{\mathrm{d} t} \sin \theta & =-\frac{\partial E}{\partial \theta} .
\end{aligned}
$$

The classical action for the single-domain chain corresponding to equation (13) is of the form:

$$
I=N \int \mathrm{d} t\left(\hbar S \frac{d \phi}{d t} \cos \theta-E(\theta, \phi)\right)
$$

where $N$ is the number of $\mathrm{A}-\mathrm{H}$ groups in the chain. The anisotropy energy

$$
E(\theta, \phi)=J S^{2} \sin ^{2} \theta-\hbar \Omega S \sin \theta \cos \phi+\frac{\hbar^{2} \Omega^{2}}{4 J}
$$

has two minima: $\boldsymbol{S}_{0}=S\left(\sin \theta_{0}, 0, \cos \theta_{0}\right)$ and $\boldsymbol{S}_{0}^{\prime}=S\left(\sin \theta_{0}, 0,-\cos \theta_{0}\right)$ (here $\left.\theta_{0}=\hbar \Omega / 2 J S\right)$, which determine two equilibrium chain orientations with opposite directions.

Thus, our problem is reduced to the model of a one-domain ferromagnetic particle with anisotropy energy given by equation (12) or equation (15). The problem of the macroscopic quantum coherence which corresponds to the tunnel switching of the particle magnetic momentum between the two equilibrium directions with the same energy was considered in detail (see, e.g., [25,26,28]), and we make use of these results to estimate the tunnelling frequency for our case.

\section{Coherent tunnel reorientation}

Since the tunnel splitting $\hbar \Delta$ for the model given by the anisotropy energy, equation (15), was calculated in [28], there is no need in repeating these calculations 
in the present paper. In this part, following [28], we dwell upon the key points of these calculations.

In order to estimate the tunnelling rate $\hbar \Delta$ of the chain transition between states $\boldsymbol{S}_{0}$ and $\boldsymbol{S}_{0}^{\prime}$ we make use of the quasi-classical (WKB) approximation that can be obtained in the instanton method framework (see, e.g., [28]). The tunnelling rate or the energy splitting is given by an expression of the type:

$$
\hbar \Delta=b \hbar \omega_{p}\left(\frac{I_{E}}{2 \pi \hbar}\right)^{\frac{1}{2}} \exp \left(-\frac{I_{E}}{\hbar}\right),
$$

where $\omega_{p}$ is oscillation frequency in the well (or small-angle precession),$b$ is a dimensionless prefactor that can be of the order 10, and $I_{E}$ is the Euclidean action for the subbarrier rotation of $\boldsymbol{S}$. In our case $I_{E}$ has the form:

$$
I_{E}=N \int \mathrm{d} \tau(-i \hbar S \phi \cos \theta+E(\phi, \theta)) \text {, }
$$

where $\tau=i t$, and the extremum of $I_{E}$ is determined by the solution of the equations

$$
\begin{aligned}
i \hbar S \frac{\mathrm{d} \theta}{\mathrm{d} \tau} \sin \theta & =\frac{\partial E}{\partial \phi}, \\
i \hbar S \frac{\mathrm{d} \phi}{\mathrm{d} \tau} \sin \theta & =-\frac{\partial E}{\partial \theta}
\end{aligned}
$$

The subbarrier trajectory corresponding to the switching of $\boldsymbol{S}$ from $\boldsymbol{S}_{0}$ at $\tau=-\infty$ to $\boldsymbol{S}_{0}^{\prime}$ at $\tau=\infty$ is the instanton solution of equation (18

$$
\begin{aligned}
\cos \theta & =-\cos \theta_{0} \tanh \left(\omega_{p} \tau\right) \\
\sin \phi & =\frac{i}{2} \frac{\cot ^{2} \theta_{0} \sec h^{2}\left(\omega_{p} \tau\right)}{\left[1+\cot ^{2} \theta_{0} \sec h^{2}\left(\omega_{p} \tau\right)\right]^{\frac{1}{2}}},
\end{aligned}
$$

where $2 \omega_{p}=\hbar \Omega \cot \theta_{0}$ is small oscillation frequency in the well. Calculating the action for this trajectory we obtain

$$
\frac{I_{E}\left(\cos \theta_{0}\right)}{\hbar}=2 S N\left[-\cos \theta_{0}+\frac{1}{2} \ln \left(\frac{1+\cos \theta_{0}}{1-\cos \theta_{0}}\right)\right] .
$$

The tunnelling rate in the quasi-classical approach is determined by the WKB exponent (depending only on $I_{E}$ ) and the prefactor, which was calculated in [28]. Using these results one can write the energy splitting $\hbar \Delta$ for the orientation transitions of a one-domain hydrogen bonded chain as

$$
\hbar \Delta=8 J S\left[\frac{S N}{\pi}\right]^{\frac{1}{2}}\left(\frac{x^{5}}{1-x^{2}}\right)^{\frac{1}{2}}\left(\frac{1-x}{1+x}\right)^{\frac{x}{2}} \exp \left(-\frac{I_{E}(x)}{\hbar}\right),
$$

where $x=\cos \theta_{0}$. 


\section{Discussion}

We can see from equations (20) and (21) that the tunnelling rate significantly depends on the atom number $N$ of a hydrogen bonded chain. But for a short $\mathrm{H}-$ bonded chain this parameter is not large. Then the tunnelling rate is not very small and the spontaneous repolarization is possible. Reference [8] gives the following estimations for the parameters of the model $J$ and $\Omega_{r}$ : the value $J / \hbar=6410$ $\mathrm{cm}^{-1}$ corresponds to the energy of D- and L-defect $\left(U / \hbar=6410 \mathrm{~cm}^{-1}\right.$ and $V / \hbar=$ $6410 \mathrm{~cm}^{-1}$ ) found in [30]; the pseudotunnel orientational frequency changes in the range $0 \leqslant \Omega_{r} \leqslant 2500 \mathrm{~cm}^{-1}$ (see [8]). Setting $\Omega_{r}=250 \mathrm{~cm}^{-1}$ and $N=5$, we obtain $\Delta \approx 5.1 \times 10^{9} \mathrm{sec}^{-1}$, while for $\Omega_{r}=50 \mathrm{~cm}^{-1}$ the tunnel frequency is $\approx 1.6 \times 10^{6} \mathrm{sec}^{-1}$, for the same $N$. For typical material parameters, the rate of macroscopic quantum tunnelling in a ferromagnetic particle is known to be of the order $10^{6} \div 10^{8} \mathrm{sec}^{-1}[25]$. Comparing this with our result, we can expect that at a low temperature the chain orienation tunnels between the two opposite directions. We can estimate critical temperature $T_{c}$, which corresponds to a crossover from the thermal to the quantum regime, i.e., the regime where reorientation frequency does not depend upon temperature (see [25]). The probability of switching the chain polarization vector due to thermal activation is proportional to $\exp \left(-\frac{\Delta E}{k_{\mathrm{B}} T}\right)$, where $\Delta E=N J S\left(1-\sin \theta_{0}\right)^{2}$ is a barrier height. Comparing it with the tunnelling probability

$$
\exp \left(-\frac{I_{E}}{\hbar}\right) \approx \exp \left(-\frac{\Delta E}{k_{\mathrm{B}} T_{c}}\right)
$$

we obtain critical temperature $T_{c}$ :

$$
T_{c} \approx \frac{\hbar \Delta E}{k_{\mathrm{B}} I_{E}}
$$

The value of $T_{c}$ has the order of $156.5 \mathrm{~K}$ for $\Omega_{r}=250 \mathrm{~cm}^{-1}$ and of $103.8 \mathrm{~K}$ for $\Omega_{r}=50 \mathrm{~cm}^{-1}$. .

Using the simplest model of protons' motion around heavy ions in a hydrogen bonded chain we have considered a possible mechanism of the tunnel oscillations of the chain polarization. This mechanism is based on the coherent tunnel motion of protons around heavy atoms and is additional to the other one realized through the coherent motion of protons along the hydrogen bonds, as described in the previous work [26]. In our study of the coherent tunnel reorientation we neglected the effects of dissipation and interaction with vibrations of a heavy sublattice. These interactions can play a significant role in tunnelling processes (see, e.g., [5,31]). The vibrations of A...A bonds lead to the modulation of a two-well proton potential and, consequently, to the renormalization of the model parameters $\Omega_{0}, \Omega_{r}$, and $J$.

\section{References}

1. G.Raimbault, F. Romain and A. Lautie, J.Raman Spectrosc. 23, 147 (1992)

2. B.V. Merinov, A.I. Branov, L.A. Shuvalov, Crystallografiya 35, 355 (1990) 
3. M. Kamoun, V. Halouani, A. Daoud, Phase Transitions 9, 327 (1987)

4. A.Matczuk, L. Kulak, and C. Bojarski, Phys. Rev. B 50, 777 (1994)

5. R. Blinc and B. Zeks, Soft Modes in Ferroelectrics and Antiferroelectrics, North-Holland Publ. Comp. - Amsterdam, Oxford American Elsevier Publishing Company, Inc. New York 1974

6. V. Ya. Antonchenco, A.S. Davydov, A.Z. Zolotariuk, Phys. Status solidi (b) 115, 631 (1983)

7. A.S. Davydov, Solitons in Molecular Systems, Reidel, Dortrecht 1986

8. I.V Stasyuk, O.L. Ivankiv, N.I. Pavlenko, J. Phys. Studies 1, 418 (1997)

9. D. Eisenberg, W. Kauzman, Structure and Properties of Water ( in russian, Leningrad, 1975)

10. G. Zundel, J. Mol. Struct. 322, 33 (1994)

11. V.V. Krasnoholovets, V.B. Taranenko, P.M. Tomchuk, and M.A. Protsenko, J. Mol. Struct. 355, 219 (1995)

12. M. Eckert and G. Zundel, J. Phys. Chem. 92, 7016 (1988)

13. B. Brzezinski and G. Zundel, Chem. Phys. Lett. 178, 138 (1991)

14. B. Brzezinski, H. Maciejevska, G. Zundel, and R. Kramer, J. Phys. Chem. 94, 528 (1990)

15. F. Bartl, G. Deckrs-Hebestreit, K. Altendorf and G. Zundel, Biophys. J. 68, 104 (1995)

16. G. Zundel, J. Mol. Struct. 381, 23 (1996)

17. W. Saenger, HHBR, XII Conf.-Workshop, Austria, Sept. (1997)

18. P.M. Tomchuk, V.V. Krasnoholovets, N.A. Protsenko, BBA 807, 272 (1985) V.V. Krasnoholovets, P.M. Tomchuk, Int. J. Quantum Chem. 33, 349 (1988)

19. A.V. Zolotaryuk, A.V. Savin, E.N. Economou, Phys. Rev. Lett. 73, 2871 (1994)

20. A.I. Sergienko, Phys. Status solidi (b) 144, 471 (1987)

21. O.E. Yanovskii, E.S. Kryachko, Phys. Status solidi (b) 147, 69 (1988)

22. A.J. Leggett et al., Rev. Mod. Phys. 59, 1 (1987)

23. S. Washburn, R.A. Webb and S.M. Faris, Phys. Rev. Lett. 54, 2712 (1985)

24. J.M. Martinus, M.M. Devoret and J.E. Lukens, Phys. Rev. Lett. 55, 1547 (1985)

25. E.M. Chudnovsky, L. Gunther, Phys. Rev. Lett. 60, 661 (1988)

26. P.M. Tomchuk, V.V. Krasnoholovets, J. Mol. Struct. 416, 161 (1997)

27. C.P. Bean, J.D. Livingston, J. Appl. Phys. 30, 120S (1959)

28. A. Garg and G.-H. Kim, Phys. Rev. B 45, 12921 (1992)

29. L. Weil, J. Chem. Phys. 51, 715 (1954)

30. R. Hassan, E. Campbell, J. Chem. Phys. 97, 4326 (1992)

31. P. Politi, A. Rettori, F. Hartmann-Boutrn, and J. Villain, Phys. Rev. Lett. 75, 537 (1995) 


\title{
Когерентна тунельна переполяризація ланцюжка водневих зв'язків
}

\author{
П.М.Томчук, С.П.Лук'янець \\ Інститут фізики НАН України, 252650 м.Київ, просп. Науки, 46
}

Отримано 24 квітня 1998 р.

В роботі запропоновано можливий механізм переполяризації короткого ланцюжка водневих зв'язків. Цей механізм базується на когерентній тунельній переорієнтації йонних груп і $є$ додатковим до механізму когерентної тунельної переполяризації водневих зв'язків, який був досліджений у попередній роботі (J.Mol.Struct. 416,161 (1997)). Частота цих тунельних переходів була отримана в квазікласичному наближенні, використовуючи інстантонний метод. Такі осциляції можуть спостерігатися при низьких температурах і їх частота не залежить від останньої, у випадку коли тунельні процеси домінують над термічно активованими процесами.

Ключові слова: водневий зв'язок, макроскопічне квантове тунелювання, макроскопічна квантова когерентність, пренос протона

PACS: $64.60 . \mathrm{Cn}, 66.30 . \mathrm{Lw}$ 
